\title{
Модель формирования операционных знаний для экспертной системы обеспечения запасными частями, инструментами и принадлежностями радиоэлектронных средств
}

\author{
С.В. Игнатьев ${ }^{1}$, д.т.н., профрессор, начальник кафедры
}

А.В. Красников ${ }^{1}$, к.т.н., доиент

A.A. Осипов ${ }^{1}$, к.m.н., преподаватель, alexalexоsipov@mail.ru

H.В. Шаталина 1, соискатель, преподаватель, nshatalina@rambler.ru

1 Ярославское высшее военное училище противовоздушной обороны, кафредра автоматики и вычислительных средств, г. Ярославль, 150001, Россия

В статье обоснована причина формирования знаний как способа их получения для экспертной системы обеспечения запасными частями, инструментами и принадлежностями радиоэлектронных средств. В соответствии с классификацией определен вид знаний как операционных.

Выявлено, что для рассматриваемой предметной области такие знания находятся на системном уровне в виде закономерностей между параметрами элементов системы обеспечения: обслуживающим персоналом, радиоэлектронными средствами, транспортной системой, базами хранения различного уровня иерархии, ремонтными и производственными предприятиями. Для их получения применяется метод формирования знаний путем разработки специализированного математического аппарата, который базируется на моделировании и выявлении требуемых закономерностей, составляющих основу новых знаний.

Основные положения работы связаны с построением модели формирования операционных знаний для экспертной системы обеспечения запасными частями, инструментами и принадлежностями радиоэлектронных средств. На первом этапе определяются структура и параметры модели. На втором этапе разрабатывается формализованное описание радиоэлектронных средств, баз хранения, ремонтного предприятия, производственного предприятия с учетом параметров обслуживающего персонала и транспортной системы. На третьем этапе осуществляется синтез формализованного описания элементов системы обеспечения в единую модель и определяется подход к получению исходных данных для формирования операционных знаний экспертной системы.

Результатом проделанной работы является программный продукт «Инструментальная среда формирования операционных знаний для экспертной системы обеспечения ЗИП радиоэлектронных систем».

Ключевые слова: экспертная система, операционные знания, модель формирования знаний, радиоэлектронные средства, система обеспечения запасными частями, инструментами и принадлежностями.

Для каждой предметной области характерны свои знания, которые являются источником для создания таких сложных программных комплексов, как экспертные системы (ЭС). Такие системы искусственного интеллекта частично заменяют специалиста определенного направления и предназначены в помощь пользователю для поиска решения в сложных ситуациях. Их разработкой занимается группа специалистов, как правило, включающая инженеров по знаниям, экспертов и программистов [1-3].

Основным направлением исследований авторов данной работы является формирование операционных знаний для ЭС в конкрет- ной предметной области. Анализ литературы [4-6] на тему возможных способов получения знаний показал отсутствие условного разграничения понятий извлечения, приобретения и формирования знаний как способа их получения.

В работах $[6,7]$ получение знаний предполагает участие эксперта, что связано с динамичностью и неполнотой данных, используемых для решения задач. При формировании закономерностей могут учитываться оценки сходства (полезности) рассматриваемых и существующих моделей сущностей через обобщенную близость путем использования интегрированной модели, что, по мнению авторов 
данной статьи, является более точным определением понятия формирования знаний. Однако чаще всего в качестве источника знаний выступает эксперт-специалист предметной области. При таком подходе со стороны инженера по знаниям возможна неточная интерпретация полученных знаний в силу неправильно выбранных методов их извлечения. Например, в статье [8] авторы отмечают, что на этапе непосредственного взаимодействия инженера по знаниям и эксперта происходит потеря извлекаемых знаний до 60 \%. В качестве альтернативного подхода приведен пример применения зарубежных и отечественных систем приобретения знаний (OMIS, OPAL, AРИАДНА и др.), работа которых, по мнению авторов, также малоэффективна в решении проблемы потерь экспертной информации. В рассматриваемой методике предлагается использовать соответствие между классами задач и методами извлечения знаний.

Анализ существующего научно-методического аппарата показывает, что наиболее сложными и малоизученными являются вопросы именно формирования знаний. Кроме того, каждая предметная область имеет свои особенности, которые дополнительно усложняют этот процесс. Так, в [9] автор разъясняет причины, по которым, согласно предметной области, ЭС должна реализовывать возможность организации единого информационного пространства для обмена предложениями среди экспертов. В основном исследования ведутся в области представления знаний.

Например, существуют такие предметные области, в которых практически отсутствуют специалисты требуемого уровня. Рассмотрим подобную ситуацию на примере системь обеспечения (СО) запасными частями, инструментами и принадлежностями (ЗИП) радиоэлектронных средств (РЭС). Данная система состоит из отдельных элементов (РЭС, базы хранения (БХ), производственные и ремонтные предприятия, обслуживающий персонал), а также транспортной системы, на которой они размещены. На сегодняшний день специалисты-эксперты в области РЭС, БХ, производственных и ремонтных предприятий имеются. Однако указанные предметные области настолько широки, что правильнее будет говорить о наличии большого количества узконаправленных экспертов. Это, конечно, вносит определенные трудно- сти в создание базы знаний, но они незначительны по сравнению с отсутствием специалистов-экспертов в области взаимодействия элементов СО ЗИП в целом как системы. Таким образом, требуются дополнительные исследования в области получения знаний.

В данной ситуации применяются методы формирования знаний на моделях (модельные эксперименты) $[10,11]$. Они основаны на разработке специального математического аппарата, который имитирует поведение реальных объектов, процессов или систем. На нем проводится серия вычислительных экспериментов для выявления требуемых закономерностей, составляющих основу новых (отсутствующих) знаний. Согласно классификации [12], подобные закономерности относятся к операционным знаниям и представляют собой общие зависимости между фактами (параметрами), которые позволяют интерпретировать данные и извлекать из них информацию. Для получения таких закономерностей необходима модель формирования операционных знаний для ЭС обеспечения ЗИП РЭС, реализованная в виде программного продукта [13].

Представим модель, которая, по мнению авторов, обеспечит получение требуемых знаний для ЭС в виде различных закономерностей:

$$
\begin{aligned}
& K_{\Gamma}=f\left(\left\{T_{\mathrm{O}_{1}}, \ldots, T_{\mathrm{O}_{n_{э 3}}}\right\}, n_{\text {эз }}, n_{\text {доп. }},\left\{T_{\text {пнэ }}, \ldots, T_{\text {пнэ }}\right\},\right.
\end{aligned}
$$

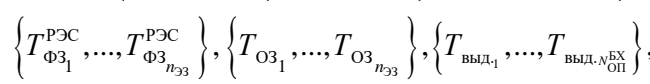

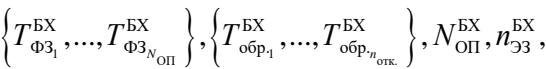

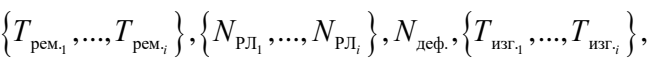

$$
\begin{aligned}
& \left\{N_{\text {Пл }_{1}}, \ldots, N_{\text {пл }}\right\}, T_{\text {ПЗ }}, T_{\text {тр. }} \text {. }
\end{aligned}
$$

С учетом ГОСТ 27.507-2015 и ГОСТ РВ 27.3.03-2005, исследований $[14,15]$ и на основании анализа функционирования СО ЗИП РЭС определены структура и параметры ее модели, а также функционал модели (1), где $K_{\Gamma}-$ коэффициент готовности; $T_{\mathrm{O}}-$ средняя наработка между отказами; $n_{\text {эз }}$ количество элементов замены (ЭЗ) РЭС; $n_{\text {доп. }}-$ минимально допустимое количество ЭЗ, при котором РЭС находится в работоспособном состоянии; $n_{\text {отк. }}-$ количество отказавших ЭЗ в РЭС;

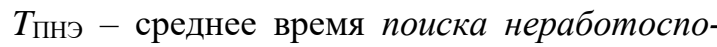
собных элементов (ПНЭ); $T_{\Phi 3}^{\text {Рэс }- \text { время } ф о р-~}$ мирования заявки (ФЗ); $T_{П 3}-$ время передачи заявки; $T_{\mathrm{O}}$ - время оформления заявки (О3) на получение работоспособного Э3; $T_{\text {обр. }}^{\text {БХ }}$ 
время обработки заявки в БХ; $n_{\ni 3}^{\text {БХ }}$ - количество находящихся на хранении Э3; $T_{\Phi 3}^{\text {Бх }-}$ время ФЗ на получение работоспособных ЭЗ в БХ; $T_{\text {выд. }}-$ время выдачи работоспособного ЭЗ в БХ; $N_{\text {ОП }}^{\text {БХ }}$ количество обслуживающего персонала (ОП) БХ; $T_{\text {тр. }}$ - время транспортировки работоспособного ЭЗ в эксплуатирующее подразделение (БХ); $N_{\text {деф. }}-$ количество отбракованных (не прошедших дефектацию) в ремонтной организации Э3; $N_{\text {Рл }}-$ количество ремонтных линий; $T_{\text {рем. }}-$ время ремонта Э3; $T_{\text {изг. }}$ - продолжительность производственного цикла изготовления Э3; $N_{\text {Пл }}$ - количество производственных линий.

Поскольку перечисленные параметры СО ЗИП являются параметрами структурных элементов системы, построение модели системы целесообразно выполнять в несколько этапов.

\section{Формализованное описание РЭС}

Источником заявок на получение работоспособных ЭЗ в СО ЗИП выступают РЭС. С учетом задач моделирования основными составляющими РЭС являются ЭЗ и обслуживающий персонал с параметрами: $n_{\ni 3}, T_{\mathrm{O}}$,

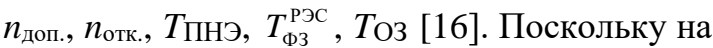
практике РЭС содержит $M$ типов ЭЗ в количестве $n_{i}$ для $i$-го типа, $i=\overline{1, M}$, состав РЭС можно описать кортежем

$$
N_{э_{3}}=\left\langle n_{{ }_{3_{1}}}, n_{э_{2}}, \ldots, n_{э_{i}}, \ldots, n_{э_{3_{M}}}\right\rangle
$$

и представить совокупностью элементов со своими показателями надежности (рис. 1). В рассматриваемом случае в качестве показателя надежности ЭЗ будем использовать среднюю наработку между отказами ( $\left.T_{\mathrm{O}}\right)$.

В силу своих конструктивных особенностей (элементной базы, монтажа) ЭЗ имеют различные значения $T_{\mathrm{O}_{i}}, i=\overline{1, M}$. Учитывая, что отказ ЭЗ является случайным событием, время его наступления тоже будет случайным с математическим ожиданием $m$ и средним квадратическим отклонением б. На практике время возникновения отказов ЭЗ может подчиняться различным законам распределения. Результаты исследований, представленные в $[15,16]$, показывают, что для отказов Э3 РЭС характерны следующие законы распределения: нормальный, гамма-распределение, экспоненциальное распределение, распределение Вейбулла, биномиальное распределение и распределение, обратное экспоненциальному. Указанные законы распределения будем использовать для формирования наработки между отказами. В свою очередь, вид и параметры закона распределения могут быть определены на основе обработки статистических данных результатов эксплуатации РЭС или их моделирования $[15,17]$.

Представим ЭЗ РЭС (рис. 1) множеством формирователей наработки между отказами с заданным законом распределения $g_{i}$, математическим ожиданием $m_{i}$ и средним квадратическим отклонением $\sigma_{i}$ (рис. 2):

$$
\begin{aligned}
& g_{i}\left(m_{i}, \sigma_{i}\right), j=\overline{1, n_{i}}, i=\overline{1, M}, \\
& G_{i}=\left\{g_{i}^{1}, g_{i}^{2}, \ldots, g_{i}^{j}, \ldots, g_{i}^{n_{i}}\right\} .
\end{aligned}
$$

Тогда формирование значения средней наработки между отказами для $j$-го ЭЗ $i$-го типа можно представить выражением

$$
T_{\mathrm{O}_{i}}^{j}=g_{i}^{j}\left(m_{i}, \sigma_{i}\right) \text {. }
$$

Такой подход позволяет моделировать отказы ЭЗ, входящих в состав РЭС, с учетом их количества, типа и надежностных характеристик.

После обнаружения отказа обслуживающий персонал с помощью средств технического диагностирования осуществляет ПНЭ. В силу разнообразных причин это время может быть различным как для разнотипных, так и для однотипных Э3 [15]. Поэтому время ПНЭ в общем случае является случайной величиной, для определения характеристик которой необходимы статистические данные. Анализ работ $[15,17]$ показал, что на практике получить такие статистические данные для существующих образцов РЭС затруднительно, поэтому

Fig. 1. A composition of radioelectronic facilities 


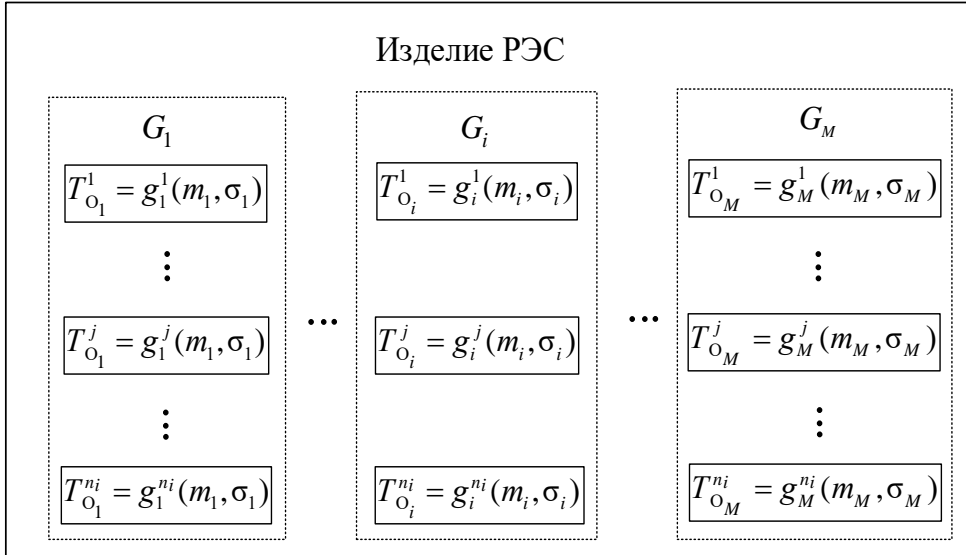

Рис. 2. Представление РЭС совокупностью формирователей наработки между отказами

Fig. 2. A representation of radioelectronic facilities as a set of formers of operating time between failures

$T_{\text {ПнЭ }}=g_{\text {ПнЭ}}\left(m_{\text {ПнЭ}}, \sigma_{\text {ПнЭ}}\right),(4)$

$T_{\mathrm{O} 3}=g_{\mathrm{O} 3}\left(m_{\mathrm{O} 3}, \sigma_{\mathrm{O} 3}\right)$.

Поскольку РЭС в СО ЗИП является источником заявок, время ФЗ может быть представлено суммой средней наработки между отказами, времени ПНЭ и времени ОЗ (рис. 3):

$T_{\text {Ф3 }_{i}}^{\text {РЭС }}=T_{\mathrm{O}_{i}}+T_{\text {пнЭ }}+T_{\text {Оз }}$.

На довольствии в БХ могут одновременно находиться несколько изделий РЭС. Для учета их количества при расчетах ЗИП в обозначениях переменных в модели изделия добавим нижний индекс $s$. Например, формирователь

при расчетах показателей надежности ис-

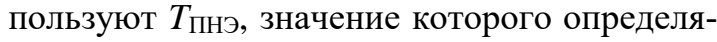
ется исходя из среднего времени восстановления изделия. Опыт эксплуатации РЭС и исследования показывают, что оно составляет примерно $80 \%$ от времени восстановления при наличии элемента в ЗИП $[13,15]$.

После определения неработоспособного Э3 обслуживающий персонал оформляет заявку на получение работоспособного ЭЗ из БХ.

В дальнейшем при описании обслуживающего персонала и настройке модели РЭС будем оперировать средним значением времени

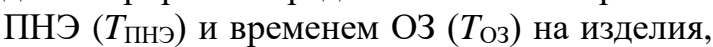
характеристики которых (закон распределения $g$, математическое ожидание $m$, среднее квадратическое отклонение $\sigma$ ) известны: наработки между отказами первого ЭЗ третьего типа второго изделия РЭС будет описываться выражением

$$
T_{\Phi 3_{3}}^{1}=g_{3_{2}}^{1}\left(m_{3_{2}}, \sigma_{3_{2}}\right) \text {. }
$$

Для описания условия перехода РЭС в неработоспособное состояние учтем, что в эксплуатационной документации изделия определен набор минимально допустимых работоспособных ЭЗ каждого типа, обеспечивающих применение РЭС по назначению с заданным качеством. Запишем набор минимально допустимых работоспособных Э3 каждого типа в виде кортежа:

$$
N_{\text {доп. }}=\left\langle n_{\text {доп. } .1}, n_{\text {доп. } 2}, \ldots, n_{\text {доп. }}, \ldots, n_{\text {доп. } .}\right\rangle \text {. }
$$

Тогда условие перехода РЭС в неработоспособное состояние (критерий отказа)

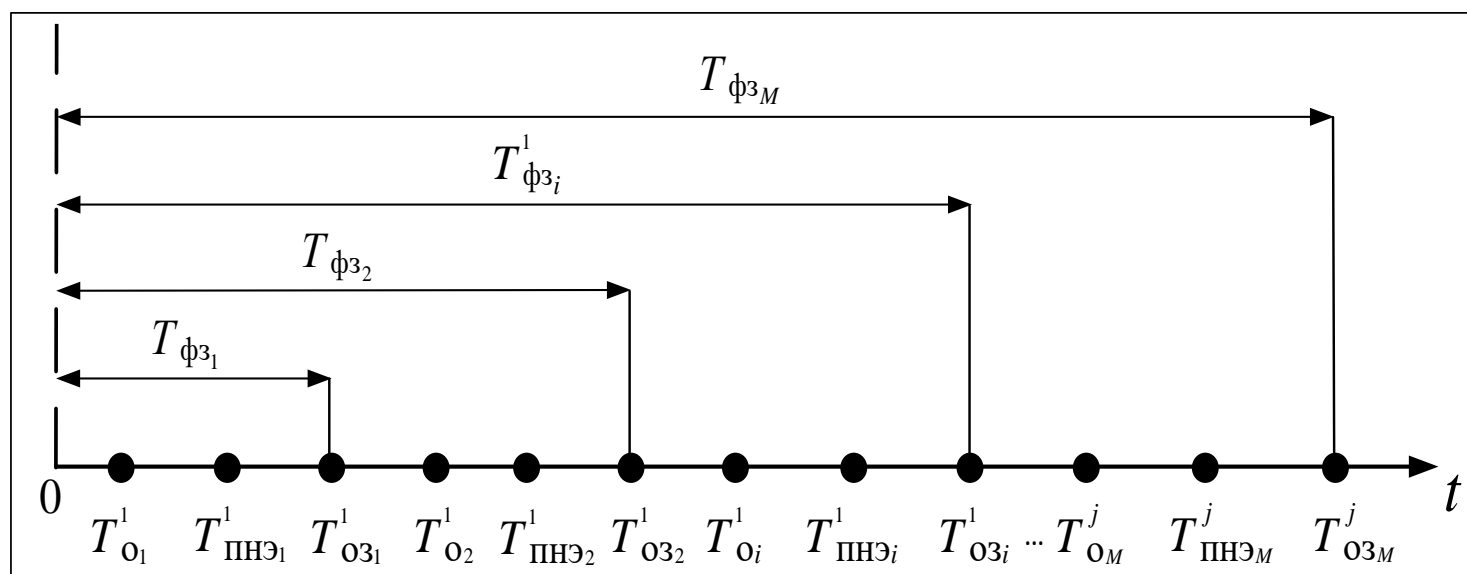

Рис. 3. Пример прочесса ФЗ на получение работоспособных ЭЗ

Fig. 3. An example of the process of generating applications for obtaining operative substitute items 
можно сформулировать следующим образом: РЭС перейдет в неработоспособное состояние в том случае, если количество работоспособных ЭЗ хотя бы одного типа в текущий момент времени $t^{\prime}$ будет меньше допустимого.

Представим состояние РЭС кортежем отказавших ЭЗ:

$$
\begin{aligned}
& N_{\text {отк. }}\left(t^{\prime}\right)=\left\langle n_{\text {отк. }}\left(t^{\prime}\right), n_{\text {отк. }}\left(t^{\prime}\right), \ldots,\right. \\
& \left.n_{\text {отк. }}\left(t^{\prime}\right), \ldots, n_{\text {отк. }}\left(t^{\prime}\right)\right\rangle,
\end{aligned}
$$

Тогда работоспособному состоянию РЭС будет соответствовать условие

$$
\left[N_{\text {эз }}-N_{\text {отк. }}\left(t^{\prime}\right)\right] \geq N_{\text {доп. }},
$$

где под разностью кортежей будем понимать операцию, аналогичную операции разности векторов:

$$
\begin{aligned}
& N_{\text {э3 }}-N_{\text {ОтК }}\left(t^{\prime}\right)= \\
& =\left\langle n_{\ni 3_{1}}, n_{\ni 3_{2}}, \ldots, n_{\ni 3_{i}}, \ldots, n_{\ni 3_{M}}\right\rangle- \\
& -\left\langle n_{\text {отк. }}\left(t^{\prime}\right), \ldots, n_{\text {отк. }_{i}}\left(t^{\prime}\right), \ldots, n_{\text {отк. } M}\left(t^{\prime}\right)\right\rangle= \\
& =\left\langle n_{\text {эз }_{1}}-n_{\text {отк. }}\left(t^{\prime}\right) ; \ldots ; n_{\text {Эз }_{i}}-n_{\text {отк }}\left(t^{\prime}\right) ; \ldots ;\right. \\
& \left.n_{\text {Эз }_{M}}-n_{\text {отк. } M}\left(t^{\prime}\right)\right\rangle ;\left[n_{\text {Эз }_{i}}-n_{\text {отк }}\left(t^{\prime}\right)\right] \geq n_{i_{\text {доп. }}} ; \\
& \overline{i=1, M} \text {. }
\end{aligned}
$$

В этом случае отказ ЭЗ не приводит к ухудшению показателей надежности РЭС (коэффициент готовности, среднее время наработки между отказами). Если в выражении (11) хотя бы по одной координате условие «больше или равно» не выполняется, то есть справедливо условие «меньше» хотя бы по одной координате, то данная ситуация характеризует отказ РЭС, что приводит к ухудшению показателей надежности. Запишем это в следующем виде (критерий отказа РЭС):

$$
\left[N_{\text {эз }}-N_{\text {отк. }}\left(t^{\prime}\right)\right]<N_{\text {доп. }},
$$

где условие «<» справедливо, если

$$
\begin{aligned}
& {\left[n_{\text {эз }}-n_{\text {отк. }_{1}}\left(t^{\prime}\right)\right]<n_{\text {доп. }} \vee \ldots . \vee\left[n_{\text {эз }_{i}}-n_{\text {отк. }_{i}}\left(t^{\prime}\right)\right]<} \\
& <n_{\text {доп. }_{i}} \vee \ldots \vee\left[n_{\text {эз }_{M}}-n_{\text {отк. }_{M}}\left(t^{\prime}\right)\right]<n_{\text {доп. }_{M}} .
\end{aligned}
$$

В формализованном виде модель группировки РЭС с учетом номенклатуры функционально необходимых элементов может быть описана следующей системой:
(Изделия РЭС

$N_{Э 3_{s}}=\left\langle n_{{ } 3_{s}}, n_{{ }_{33_{s}}}, \ldots, n_{{ }_{3 i_{s}}}, \ldots, n_{\ni 3_{M_{s}}}\right\rangle$.

Формирование заявок на ЗИП

$G_{i_{s}}=\left\{g_{i_{s}}^{1}, g_{i_{s}}^{2}, \ldots, g_{i_{s}}^{i_{i_{s}}}\right\}, s=\overline{1, S ;} i_{s}=\overline{1, M_{s}} ;$

$T_{\mathrm{O}_{i_{s}}}=g_{i_{s}}^{n_{i_{s}}}\left(m_{i_{s}}, \sigma_{i_{s}}\right)$;

$T_{\text {Пнэ }}=g_{\text {Пнэ }}\left(m_{\text {Пнэ }}, \sigma_{\text {Пнэ }}\right)$;

$T_{\mathrm{\Phi} 3 i s_{s}}=T_{\mathrm{O}_{i_{s}}}+T_{\text {пнЭ }}+\mathrm{T}_{\mathrm{O}_{s}}$,

$s=\overline{1, S ;} i=\overline{1, M_{s}}$.

Формирование наработки между

отказами изделий РЭС: количество

допустимых Э3, обеспечивающих

$F=$

работоспособное состояние изделий

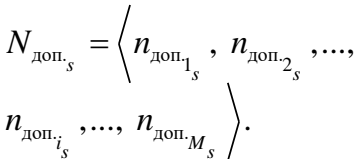

Количество откзавших ЭЗ

$N_{\text {отк }_{\text {s }}}(t)=\left\langle n_{{\text {отк. } \text {. }_{s}}_{s}}(t), n_{\text {отк. }_{2_{s}}}(t), \ldots\right.$,
$\left.n_{\text {отк }_{i_{s}}}(t), \ldots, n_{\text {отк. }_{M_{s}}}(t)\right\rangle ;$
$n_{\text {отк }_{i_{s}}}(t)=f\left(G_{i_{s}}\right) ; i=\overline{1, M .}$

Критерий отказа изделий РЭС

$\left[N_{\text {Эз }}-N_{\text {отк. }_{s}}(t)\right]<N_{\text {доп. }}$,
$M_{s}\left[n_{\text {эз }}-n_{\text {отк. }_{i}}(t)\right]<n_{\text {доп }_{i_{s}}}$

\section{Формализованное описание БХ}

Сформированная обслуживающим персоналом РЭС заявка передается в БХ первого уровня. Передача заявки осуществляется с использованием средств доставки, в качестве которых могут выступать транспортные средства или линии передачи данных (связи), поэтому время передачи заявки ( $\left.\mathrm{T}_{\text {пз }}\right)$ получателю может варьироваться в значительных пределах.

При поступлении в БХ осуществляется обработка заявки должностными лицами, которая заключается в проверке наличия в БХ запрашиваемого работоспособного ЭЗ. Этот процесс характеризуется временным показателем - временем обработки заявки ( $\left.T_{\text {обр. }}^{\mathrm{LX}}\right)$, которое является случайной величиной с задан- 
ным законом распределения $g$, математическим ожиданием $m$ и средним квадратическим отклонением $\sigma[15,16]$. Обусловлено это тем, что у персонала БХ различные уровень подготовки, опыт работы, психофизические качества и т.д.

В процессе обработки заявки персонал БХ выполняет определенную последовательность действий (операций): ознакомление с поступившим уведомлением, проверка ведомости ЗИП, оформление документации на выдачу ЭЗ и т.д. Время выполнения этих действий в силу описанных ранее причин может быть различным. Поскольку действия по обработке поступившей заявки персоналом БХ выполняются последовательно, время обработки заявки представляет собой сумму времени выполнения указанных действий (операций). Тогда на основании центральной предельной теоремы время обработки заявки представляет собой случайную величину, распределенную по нормальному закону $g$, с математическим ожиданием $m$ и средним квадратическим отклонением $\sigma$ :

$$
T_{\text {обр. }}^{\text {БХ }}=g_{\text {обр. }}^{\text {БХ }}\left(m_{\text {обр. }}^{\text {БХ }}, \sigma_{\text {обр. }}^{\text {БХ }}\right) .
$$

В последующем такой подход применяется при формализованном описании операций, выполняемых человеком.

В случае наличия запрашиваемых ЭЗ персоналом БХ осуществляется их выдача. Время выдачи работоспособного ЭЗ ( $\left.T_{\text {выд. }}\right)-$ это время, необходимое для заполнения соответствующей документации на выдаваемые Э3 (накладные, карточки учета ЗИП, карточка некомплектности, наряд на получение материальных ценностей и др.). Время выдачи работоспособного ЭЗ, определяемое действиями персонала БХ, также будет представлять собой случайную величину, распределенную по нормальному закону $g$ с математическим ожиданием $m$ и средним квадратическим отклонением $\sigma$ :

$$
T_{\text {выд. }}^{\text {БХ }}=g_{\text {выд. }}^{\text {БХ }}\left(m_{\text {выд. }}^{\text {БХ }}, \sigma_{\text {выд. }}^{\text {БХ }}\right) .
$$

Кроме указанных параметров, время выдачи будет зависеть от количества сотрудников (персонала) БХ $\left(N_{\text {ОП }}^{\text {БХ }}\right)$.

Как правило, количество Э3, находящихся в БХ, ограничено. Представим их номенклатуру и количество кортежем

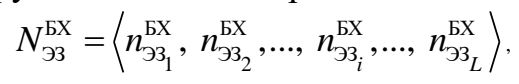

где $L$ - количество типов Э3, находящихся на хранении.
В процессе удовлетворения заявок количество ЭЗ в БХ будет меняться. Представим текущее состояние БХ, а именно наличие ЭЗ в момент времени $t^{\prime}$, кортежем

$$
\begin{aligned}
& N_{\text {тек. }}^{\mathrm{БX}}\left(t^{\prime}\right)= \\
= & \left\langle n_{\text {тек. }_{1}}^{\mathrm{БX}}\left(t^{\prime}\right), n_{\text {тек. }_{2}}^{\text {БХ }}\left(t^{\prime}\right), \ldots, n_{\text {тек }_{i}}^{\mathrm{БX}}\left(t^{\prime}\right), \ldots, n_{\text {тек. }_{L}}^{\mathrm{БX}}\left(t^{\prime}\right)\right\rangle .
\end{aligned}
$$

На практике возможны ситуации, когда при поступлении заявки в момент времени $t^{\prime}$ необходимый ЭЗ $i$-го типа в БХ отсутствует или количество запрашиваемых (требуемых) Э3 превышает количество элементов, находящихся на хранении. В этом случае возникает отказ в обслуживании и необслуженная заявка передается в БХ более высокого уровня. В формализованном виде отказу в обслуживании будет соответствовать условие

$$
\left[N_{\text {тек. }}^{\text {Бх }}\left(t^{\prime}\right)-N_{\text {отк. }}\left(t^{\prime}\right)\right]<0,
$$

где под разностью кортежей будем понимать операцию, аналогичную операции разности векторов. При этом условие «меньше» справедливо, если выполняется неравенство

$$
\begin{aligned}
& {\left[n_{\text {тек. }}^{\text {БХ }}\left(t^{\prime}\right)-n_{\text {отк. }}\left(t^{\prime}\right)\right]<0 \vee \ldots} \\
& \vee\left[n_{\text {тек. }}^{\text {БХ }}\left(t^{\prime}\right)-n_{\text {отк. }_{i}}\left(t^{\prime}\right)\right]<0 \vee \ldots . \\
& \vee\left[n_{\text {тек. }_{L}}^{\text {БХ }}\left(t^{\prime}\right)-n_{\text {отк. }}\left(t^{\prime}\right)\right]<0 .
\end{aligned}
$$

После удовлетворения поступившей заявки в БХ возникает текущий некомплект, для ликвидации которого требуется восполнить запасы. На основании ГОСТ 27.507-2015 и [15] для восполнения запасов персонал БХ в соответствии с заданной стратегией пополнения формирует заявку на получение недостающего количества работоспособных ЭЗ $i$-го типа в БХ более высокого уровня иерархии. Поскольку процесс Ф3 представляет собой последовательность действий персонала БХ, среднее время Ф3 ( $\left.T_{\Phi 3}^{\mathrm{БX}}\right)$ также является случайной величиной, распределенной по нормальному закону $g$ с математическим ожиданием $m$ и средним квадратическим отклонением $\sigma$ :

$$
T_{\Phi 3}^{\text {БХ }}=g_{\Phi 3}^{\text {БХ }}\left(m_{\Phi 3}^{\text {БХ }}, \sigma_{\Phi 3}^{\text {БХ }}\right) \text {. }
$$

Текущий некомплект в формализованном виде представим кортежем отсутствующих ЭЗ в БХ:

$$
N_{\text {orc. }}^{\mathrm{EX}}\left(t^{\prime}\right)=\left\langle n_{\text {orc. }_{1}}^{\mathrm{EX}}\left(t^{\prime}\right), n_{\text {orc. }_{2}}^{\mathrm{EX}}\left(t^{\prime}\right), \ldots, n_{\text {orc. }_{i}}^{\mathrm{EX}}\left(t^{\prime}\right), \ldots, n_{\text {orc. }}^{\mathrm{EX}}\left(t^{\prime}\right)\right\rangle,
$$

где $N_{\text {отс. }}^{\mathrm{EX}}\left(t^{\prime}\right)=N_{\text {ЭЗ }}^{\mathrm{EX}}-N_{\text {тек. }}^{\mathrm{EX}}=$

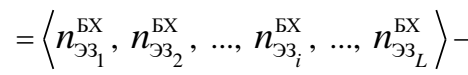

$-\left\langle n_{\text {тек. }{ }_{1}}^{\text {БХ }}\left(t^{\prime}\right), \ldots, n_{\text {тек. }_{i}}^{\text {БХ }}\left(t^{\prime}\right), \ldots, n_{\text {тек. }_{L}}^{\text {БХ }}\left(t^{\prime}\right)\right\rangle$, 


$$
\begin{aligned}
& \text { или } N_{\text {отс. }}^{\mathrm{LX}}\left(t^{\prime}\right)=
\end{aligned}
$$

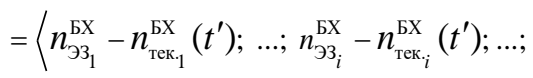

$$
\begin{aligned}
& \left.n_{{ }_{\jmath_{L}}}^{\mathrm{5X}}-n_{\text {тек. }_{L}}^{\mathrm{LX}}\left(t^{\prime}\right)\right\rangle, i=\overline{1, L} \text {. }
\end{aligned}
$$

В состав СО ЗИП, как правило, входят несколько БХ. Для их обозначения в СО ЗИП будем использовать индекс $z$. Например, $g_{\Phi 3}^{\mathrm{LX}}\left(m_{\Phi 3}^{\mathrm{LX}}, \sigma_{\Phi 3}^{\mathrm{LX}}\right)-$ формирователь времени подачи заявки на получение работоспособного ЭЗ персоналом $z$-й БХ.

\section{Формализованное описание ремонтного предприятия}

В существующей СО ЗИП предусмотрено, что неработоспособные ЭЗ поступают на ремонтное предприятие. При поступлении они проходят очистку, учет, дефектацию, после чего осуществляется восстановление их работоспособности.

Выполнение полного технологического цикла ремонта неработоспособного ЭЗ $i$-го типа характеризуется временем ремонта $\left(T_{\text {рем. }}\right)$. Поскольку процесс ремонта связан с действиями персонала ремонтного предприятия, среднее время ремонта $T_{\text {рем. }}$ является случайной величиной, закон распределения $g$, параметры $m$ и $\sigma$ которой могут быть получены на основе обработки статистических данных по ремонту ЭЗ $i$-го типа.

Таким образом, время ремонта ЭЗ $i$-го типа представим выражением

$$
T_{\text {peм. }_{i}}=g_{\text {pem. }_{i}}\left(m_{\text {pem. }_{i}}, \sigma_{\text {peм. }_{i}}\right) \text {. }
$$

Кроме указанных параметров, время ремонта будет зависеть от количества ремонт-

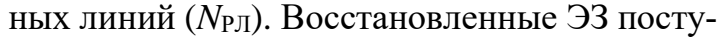
пают в БХ первого (нижнего) уровня, а по отбракованным (не прошедшим дефекта-

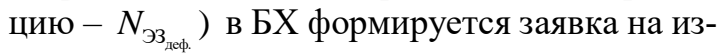
готовление новых ЭЗ. Поскольку в последнем случае текущий некомплект БХ, обусловленный изъятием ЭЗ для нужд РЭС, не может быть ликвидирован за счет ремонтного предприятия, персоналом БХ формируется заявка на пополнение ЭЗ.

Предсказать количество дефектных неработоспособных ЭЗ из всех поступивших на ремонтное предприятие заранее невозможно. Следовательно, появление таких элементов будем задавать вероятностью $P$, значение которой может быть вычислено с использованием статистических данных по ремонту ЭЗ:

$$
N_{\text {деф. }}\left(t^{\prime}\right)=\left\lceil N_{\text {отк. }}\left(t^{\prime}\right) P\right\rceil .
$$

Кроме того, на практике ремонтное предприятие выполняет восстановление ограниченного типа ЭЗ (например, это ограничение может определяться типом изделия, функциональным назначением Э3). Обозначим количество типов восстанавливаемых ЭЗ $K$. Тогда вероятность поступления дефектного неработоспособного ЭЗ от $s$-го образца РЭС представим кортежем

$$
\begin{aligned}
& P_{S}=\left\langle p_{1_{s}}, p_{2_{s}}, \ldots, p_{i_{s}}, \ldots, p_{k_{s}}\right\rangle, \\
& s=\overline{1, S}, i_{s}=\overline{1, K_{s}} .
\end{aligned}
$$

В результате на выходе ремонтного предприятия формируется поток необслуженных заявок.

Количество дефектных неработоспособных ЭЗ на выходе ремонтного предприятия в текущий момент времени $t^{\prime}$ (количество необслуженных заявок) представим кортежем

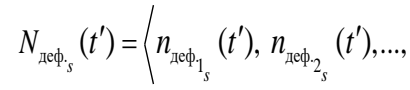

$$
\begin{aligned}
& \left.n_{\text {де中 }_{i_{s}}}\left(t^{\prime}\right), \ldots, n_{{\text {дер. } K_{s}}}\left(t^{\prime}\right)\right\rangle,
\end{aligned}
$$

где

$N_{\text {सeф. }}\left(t^{\prime}\right)=\left\lceil N_{\text {ork }_{s}}\left(t^{\prime}\right) P_{s}\right\rceil=$

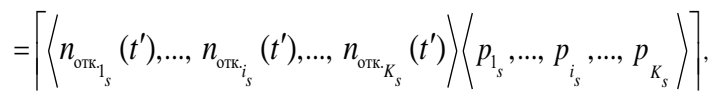

или

$N_{\text {деф. }}\left(t^{\prime}\right)=$

$=\left[\left\langle n_{{\text {OTK } \mu_{s}}_{S}}\left(t^{\prime}\right) p_{1_{s}} ; \ldots ; n_{\text {OTK }_{i_{s}}}\left(t^{\prime}\right) p_{i_{s}} ; \ldots ; n_{\text {оTK. } K_{S}}\left(t^{\prime}\right) p_{K_{s}}\right\rangle\right],(27)$

$s=\overline{1, S}, i_{s}=\overline{1, K_{s}}$.

СО ЗИП может иметь в своем составе несколько ремонтных предприятий. Для их обозначения в модели СО ЗИП введем индекс $q$. Например, $T_{\text {peм. }_{i}}^{q}=g_{\text {peм. }_{i}}^{q}\left(m_{\text {peм.. }_{i}}^{q}, \sigma_{\text {peм.. }_{i}}^{q}\right)-$ формирователь времени ремонта $i$-го ЭЗ $q$-м ремонтным предприятием.

\section{Формализованное описание производственного предприятия}

Оконечным элементом СО ЗИП является производственное предприятие, осуществляющее изготовление ЭЗ и имеющее свои параметры.

Изготовление ЭЗ осуществляется в процессе производства, который обусловлен переработкой используемых ресурсов (сырья) в полезную продукцию - ЭЗ. 
Процесс производства включает в себя совокупность выполняемых на предприятии технологических процессов и может сочетать как операции, требующие непосредственно труда со стороны производственного персонала, так и естественные процессы, в которых изменение свойств ресурсов осуществляется без участия работников [15]. Законченный круг производственных операций от первой до последней при изготовлении ЭЗ называется производственным циклом.

Показателем производственного цикла является его временная продолжительность, представляющая собой интервал времени от начала первой производственной операции до окончания последней. Обозначим это время как $T_{\text {изг. }}$.

В силу того, что ЭЗ имеют свои конструктивные особенности (элементная база, монтаж), процесс производства будет характеризоваться различным количеством выполняемых технологических операций и, следовательно, различным временем изготовления.

Поскольку процесс изготовления связан с действиями персонала производственного предприятия, среднее время изготовления $\left(T_{\text {изг. }}\right)$ является случайной величиной, закон распределения $g$, параметры $m$ и $\sigma$ которой могут быть вычислены на основе статистических данных по изготовлению ЭЗ $i$-го типа.

Следовательно, в модели СО ЗИП время изготовления ЭЗ $i$-го типа будет определяться выражением

$$
T_{\text {изг } r_{i}}=g_{\text {изг. } r_{i}}\left(m_{\text {изг }_{i}}, \sigma_{\text {изг. }_{i}}\right) \text {. }
$$

С целью повышения производительности и надежности производственного процесса при изготовлении продукции на предприятии может быть организовано параллельное производство, которое предполагает одновременное выполнение различных операций при изготовлении одного и того же ЭЗ. Такое производство реализуется за счет организации параллельно функционирующих производственных линий, оснащенных однотипным оборудованием и реализующих производственный цикл. Количество таких линий обозначим как $N_{\text {Пл. }}$.

Поскольку в состав СО ЗИП может входить различное количество производственных предприятий, для их обозначения добавим индекс $h$. Например, $T_{\text {изг. }_{i}}^{h}=$ $=g_{\text {изг } r_{i}}^{h}\left(m_{\text {изг } r_{i}}^{h}, \sigma_{\text {нзг } r_{i}}^{h}\right)-$ формирователь времени изготовления ЭЗ $i$-го типа на $h$-м производственном предприятии.

\section{Формализованное описание СО ЗИП}

Поскольку основной задачей СО ЗИП при удовлетворении заявки является доставка работоспособного элемента, временным показателем этого процесса будет выступать время доставки.

Согласно ГОСТ 27.507-2015 время доставки работоспособного ЭЗ представляет собой интервал времени от момента Ф3 на получение ЭЗ до момента его поступления к месту проведения текущего ремонта РЭС. Другими словами, время доставки представляет собой сумму временных интервалов процессов, протекающих в СО ЗИП при удовлетворении заявки [15]. Тогда время доставки Э3 $i$-го типа из БХ первого уровня может быть представлено выражением

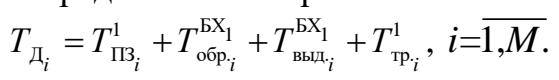

Выражение (29) справедливо в том случае, если в БХ первого уровня есть в наличии запрашиваемый ЭЗ $i$-го типа, то есть не выполняется условие (18). В противном случае возникает отказ в обслуживании. Поскольку существующая СО ЗИП имеет иерархическую структуру, заявка передается в БХ второго уровня. В результате выражение для времени доставки примет следующий вид:

$$
\begin{aligned}
& T_{\text {म }_{i}}=T_{\Pi 3_{i}}^{1}+T_{\text {об̆ }_{i}}^{\mathrm{LX}_{1}}+T_{\Phi 3_{i}}^{\mathrm{LX}_{1}}+T_{\Pi_{i} 3_{i}}^{2}+ \\
& +T_{\text {обр. }_{i}}^{\mathrm{5X}_{2}}+T_{\text {выд. }_{2}}^{\mathrm{БХ}_{2}}+T_{\text {тр }_{i}}^{2} .
\end{aligned}
$$

Для случая удовлетворения заявки БХ третьего уровня

$$
\begin{aligned}
& T_{\text {म }_{i}}=T_{\Pi_{i}}^{1}+T_{\text {обр }_{i}}^{\mathrm{EX}}+T_{\Phi 3_{i}}^{\mathrm{LX}_{1}}+T_{\Pi_{i}}^{2}+T_{\text {обр }_{i}}^{\mathrm{LX}_{2}}+
\end{aligned}
$$

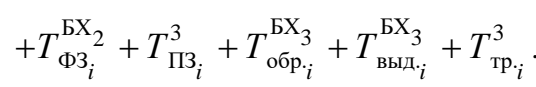

Из выражений (30) и (31) видно, что на каждом уровне иерархии можно выделить последовательность повторяющихся действий (передача, обработка, ФЗ). С алгоритмической точки зрения количество задействованных в удовлетворении заявки уровней БХ будет определять количество циклов алгоритма. Тогда время выдачи ЭЗ из БХ $z$-го уровня может быть представлено выражением

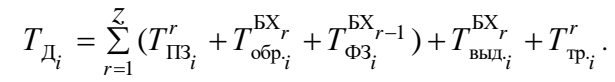

Если во всех БХ отсутствует запрашиваемый ЭЗ (условие (18) выполняется для всех 
БХ), то удовлетворение заявки осуществляется производственным предприятием и выражение (32) примет следующий вид:

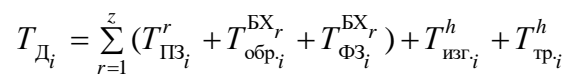

Как было отмечено ранее, после удовлетворения заявки в БХ возникает текущий некомплект.

Процесс восполнения запасов в БХ будет зависеть от структуры СО ЗИП и принятой стратегии пополнения [10]. При этом возможны следующие варианты пополнения: из вышестоящей БХ, из производственного и ремонтного предприятий.

Как и для РЭС, временем удовлетворения заявки для БХ выступает время доставки ЭЗ, которое представляет собой интервал времени от момента изъятия ЭЗ из БХ до момента поступления ЭЗ в соответствующую БХ. При пополнении запасов БХ первого уровня из БХ второго уровня время доставки будет определяться выражением

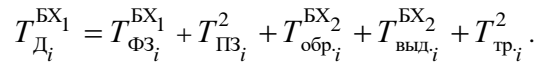

Если в БХ второго уровня запрашиваемый Э3 отсутствует (не выполняется условие (18)), то возникает отказ в обслуживании и заявка передается в БХ третьего уровня. В результате выражение для времени доставки будет следующим:

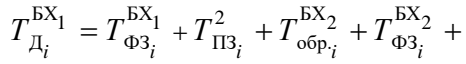

$$
\begin{aligned}
& +T_{\text {Пз }_{i}}^{3}+T_{\text {обр }_{i}}^{\mathrm{5X}_{3}}+T_{\text {выд. }_{i}}^{\mathrm{БХ}_{3}}+T_{\text {тр }_{. i}}^{3}
\end{aligned}
$$

Тогда выражение для времени доставки ЭЗ в $u$-ю БХ из БХ $r$-го уровня в общем виде может быть представлено выражением

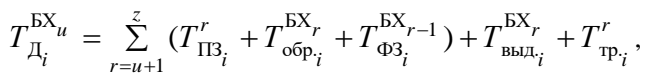

где $u=\overline{1, Z}$.

Если в БХ z-го уровня отсутствует запрашиваемый Э3, то персонал БХ формирует заявку на изготовление ЭЗ, которая передается производственному предприятию. В результате выражение (36) примет следующий вид:

$$
T_{\text {म }_{i}}^{\mathrm{EX}_{u}}=\sum_{r=u+1}^{z}\left(T_{\Pi_{i}}^{r}+T_{\text {обр. }_{i}}^{\mathrm{EX}_{r}}+T_{\Phi 3_{i}}^{\mathrm{EX}_{r}}\right)+T_{\text {изг. }_{i}}^{h}+T_{\text {тр }_{i}}^{h} .
$$

Для варианта восполнения запасов в БХ из производственного предприятия время доставки определяется выражением

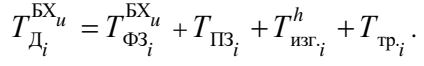

Рассмотрим вариант восполнения запасов БХ из ремонтного предприятия.

В этом случае время доставки будет определяться выражением

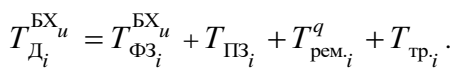

Если ремонт ЭЗ невозможен, то в БХ поступает соответствующее уведомление и восполнение запасов будет осуществляться из вышестоящей БХ или производственного предприятия.

В первом случае время доставки будет эквивалентно времени доставки заявки в РЭС (31) с учетом прохождения заявки через ремонтное предприятие:

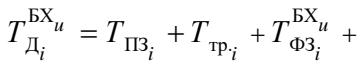

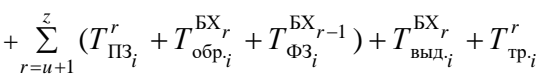

Если в БХ z-го уровня отсутствует запрашиваемый Э3, то обслуживающий персонал БХ формирует заявку на изготовление Э3, которая передается на производственное предприятие:

$$
\begin{aligned}
& T_{\text {Д }_{i}}^{\mathrm{LX}_{u}}=T_{\Pi 3_{i}}+T_{\text {Tp. }_{i}}+T_{\Phi 3_{i}}^{\mathrm{EX}{ }^{2}}+
\end{aligned}
$$

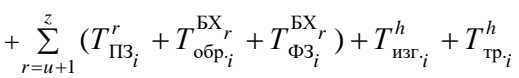

Для структуры, предусматривающей восполнение запасов непосредственно из производственного предприятия, время доставки будет определяться выражением

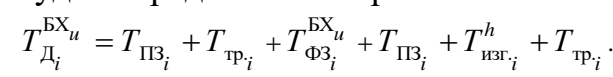

Таким образом, модель формирования операционных знаний для ЭС обеспечения ЗИП РЭС представляет собой систему взаимосвязанных выражений (13), (15)-(18), (20), (21), (23), (24), (27), (28), (30), (33), (36)-(42).

Примеры работы программы по расчету влияния показателей параметров СО ЗИП друг на друга и на показатели надежности PЭС показаны на рисунках (см. http://www. swsys.ru/uploaded/image/2021-4/2021-4-dop/ 14.jpg, http://www.swsys.ru/uploaded/image/ 2021-4/2021-4-dop/15.jpg).

\section{Заключение}

Модель реализована в виде программного продукта на языке программирования $\mathrm{Ob}$ jectPascal в среде BorlandDelphi 7.0. Получено свидетельство о регистрации программы для ЭВМ № 2021663603.

Функциональные возможности модели позволяют имитировать отказы и процессы восстановления РЭС в заданном масштабе времени, произвести расчет влияния показателей параметров системы обеспечения ЗИП друг на друга и на показатели надежности 
РЭС. Обосновав границы и шаг изменения каждого параметра этой модели, откалибровав ее для конкретной СО ЗИП РЭС и проведя полнофакторный эксперимент, в отличие от известных моделей можно получить исходные данные для формирования операционных знаний ЭС в виде требуемых закономерностей.

\title{
Лumepamypa
}

1. Пискорская С.Ю., Черненко В.В. Экспертные системы // Актуальные проблемы авиации и космонавтики: матер. VIII Всерос. науч.-практич. конф. 2012. Т. 2. № 8. С. 322-323.

2. Култыгин О.П. Экспертные системы анализа предметной области для проектирования информационных систем // Прикладная информатика. 2020. Т. 15. № 2. С. 105-118.

3. Нильсон Н. Принципы искусственного интеллекта; [пер. с англ.]. М.: Радио и связь, 1985. $375 \mathrm{c}$.

4. Захарова Е.В., Баранова Ж.М., Третьяков Е.В. Проблемы приобретения экспертной информации при создании экспертных систем и подход к их решению // Энергетика, информатика, инновации: сб. тр. IV Междунар. науч.-практич. конф. Смоленск. 2014. С. 48-54.

5. Ручкин В.М., Фулин В.А. Экспертные системы и базы знаний. Рязань, 2009. 28 с.

6. Трембач В.М. Методы формирования, использования и анализа баз знаний // Экономика, статистика и информатика. 2010. № 4. С. 145-149.

7. Трембач В.М. Приобретение знаний из опыта и внешних источников // Сб. тр. Х Национал. конф. по искусственному интеллекту с междунар. уч. 2006. Т. 3. С. 1100-1108.

8. Балашов О.В., Букачев Д.С. Выбор методов извлечения и обработки экспертной информации для базы знаний систем поддержки принятия решения // Международный журнал информационных технологий и энергоэффективности. 2018. Т. 3. № 4. С. 28-35.

9. Гурьянов А.В. Вопросы разработки информационной экспертной системы для оценки качества ЗИП // Системный анализ и логистика. 2016. № 12. С. 76-80.

10. Дмитриев П.А., Анисимов О.В., Игнатьев С.В., Богун В.В. Информационные технологии в науке и образовании. Ярославль: Изд-во ЯВВУ ПВО, 2016. 264 с.

11. Глущенко П.В. Техническая диагностика: моделирование в диагностировании и прогнозировании состояния технических объектов. М.: Вузовская книга, 2013. 247 с.

12. Телков А.Ю. Экспертные системы. Воронеж: Изд-во ИПЦ ВГУ, 2007. 83 с.

13. Цуганов С.А., Красников А.В., Осипов А.А., Шаталина Н.В., Ехилевский М.Л., Кирпичев А.М. Инструментальная среда формирования операционных знаний для экспертной системы обеспечения ЗИП радиоэлектронных систем: Свид. о регистр. ПрЭВМ № 2021663603. Рос. Федерация, 2021.

14. Черкесов Г.Н. О критериях выбора комплектов ЗИП // Надежность. 2013. № 2. С. 3-18.

15. Игнатьев С.В., Боев С.Ф., Тихонов В.Б. и др. Надежность и эксплуатация радиотехнических систем. М.: Научная книга, 2018. 370 с.

16. Половко А.М., Гуров С.В. Основы теории надежности. СПб: БХВ-Петербург, 2006. 704 с.

17. Игнатьев С.В., Плакса Ю.А., Красников А.В., Дрожжин А.В. Модель группировки радиоэлектронных систем для оценки временных показателей надежности // Программные продукты и системы. 2018. № 3. C. 598-604. DOI: 10.15827/0236-235X.123.598-604.

\section{A model for the forming operational knowledge for an expert system for providing radioelectronic facilities with spare parts, tools and accessories}

\author{
S.V. Ignatev ${ }^{1}$, Dr.Sc. (Engineering), Professor, Head of Department \\ A.V. Krasnikov ${ }^{1}$, Ph.D. (Engineering), Associate Professor \\ A.A. Osipov ${ }^{1}$, Ph.D. (Engineering), Lecturer, alexalexosipov@mail.ru \\ N.V. Shatalina ${ }^{1}$, Applicant, Lecturer, nshatalina@ rambler.ru \\ ${ }^{1}$ Yaroslavl Higher Military School of Air Defense, Department of Automation and Computing, \\ Yaroslavl, 150001, Russian Federation
}


Abstract. The paper justifies the reason for forming knowledge as a way of obtaining it for an expert system that provides radioelectronic facilities with spare parts, tools and accessories. It defines the type of knowledge as operational according to the classification.

The paper reveals that for the subject area under consideration, such knowledge is at the system level in the form of regularities between the parameters of the support system elements: service personnel, radioelectronic facilities, transport system, storage bases of various hierarchical levels, repair and production enterprises. To obtain them, the authors use the method of knowledge formation by developing a specialized mathematical apparatus based on modeling and identifying the required patterns that form the basis of new knowledge.

The main provisions of the work are related to constructing a model for forming operational knowledge for an expert system for providing radioelectronic facilities with spare parts, tools and accessories. The first stage involves determining the model structure and parameters. The second stage includes developing a formalized description of radioelectronic facilities, storage bases, a repair enterprise, a production enterprise, taking into account the parameters of the service personnel and the transport system. The third stage synthesizes a formalized description of the support system elements into a single model and determines an approach to obtaining the initial data for forming the expert system operational knowledge.

The result of the work done is the software product called the Instrumental environment for forming operational knowledge for the expert system for providing radioelectronic facilities with spare parts.

Keywords: expert system, operational knowledge, knowledge formation model, radioelectronic means, a system for providing spare parts, tools and accessories.

\section{References}

1. Piskorskaya S.Yu., Chernenko V.V. Expert systems. Proc. VIII All-Russ. Sci. Pract. Conf. Urgent Problems of Aviation and Astronautics, 2012, vol. 2, no. 8, pp. 322-323 (in Russ.).

2. Kultygin O.P. Expert analysis systems of subject area for the design of information systems. J. of Applied Informatics, 2020, vol. 15, no. 2, pp. 105-118 (in Russ.).

3. Nilsson N.J. Principles of Artificial Intelligence. CA, PaloAlto: Tioga Press Publ., 1980, 476 p. (Russ. ed.: Moscow, 1985, 375 p.).

4. Zakharova E.V., Baranova Zh.M., Tretyakov E.V. The problems of acquiring expert information when creating expert systems and an approach to their solution. Proc. IV Int. Sci. Pract. Conf. Power Engineering, Informatics, Innovation, 2014, pp. 48-54 (in Russ.).

5. Ruchkin V.M., Fulin V.A. Expert Systems and Knowledge Bases. Ryazan, 2009, 28 p. (in Russ.).

6. Trembach V.M. Methods of formation, usage and knowledge base analysis. Ekonomika, Statistika $i$ Informatika. Vestnik UMO, 2010, no. 4, pp. 145-149 (in Russ.).

7. Trembach V.M. Knowledge acquisition from experience and external sources. Proc. X National Conf. on Artificial Intelligence with Int. Participation, 2006, vol. 3, pp. 1100-1108 (in Russ.).

8. Balashov O.V., Bukachev D.S. Selection of methods for extracting and processing of expert information for the knowledge base of decision support system. Int. J. of Inf. Tech. and Energy Efficiency, 2018, vol. 3, no. 4, pp. 28-35 (in Russ.).

9. Guryanov A.V. The isses of developing an information expert system for assessing the quality of spare parts. The System Analysis and Logistics, 2016, no. 12, pp. 76-80 (in Russ.).

10. Dmitriev P.A., Anisimov O.V., Ignatev S.V., Bogun V.V. Information Technologies in Science and Education. Yaroslavl, 2016, 264 p. (in Russ.).

11. Glushchenko P.V. Technical Diagnostics: Modeling in Diagnosing and Predicting the State of Technical Objects. Moscow, 2013, 247 p. (in Russ.).

12. Telkov A.Yu. Expert Systems. Voronezh, 2007, 83 p. (in Russ.).

13. Tsuganov S.A., Krasnikov A.V., Osipov A.A., Shatalina N.V., Ekhilevskiy M.L., Kirpichev A.M. An Instrumental Environment for Forming Operational Knowledge for an Expert System for Providing Spare Parts for Radioelectronic Systems. Patent RF, no. 2021663603, 2021.

14. Cherkesov G.N. On criteria of selection of SPTA kits. Dependability, 2013, no. 2, pp. 3-18 (in Russ.). 
15. Ignatev S.V., Boev S.F., Tikhonov V.B. et al. Reliability and Operation of Radio Engineering Systems. Moscow, 2018, 370 p. (in Russ.).

16. Polovko A.M., Gurov S.V. The Reliability Theory Fundamentals. St. Petersburg, 2006, 704 p. (in Russ.).

17. Ignatev S.V., Krasnikov A.V., Plaksa Yu.A., Drozhin A.V. A nested model of radio-electronic systems for estimation of temporary reliability. Software and Systems, 2018, no. 3, pp. 598-604. DOI: 10.15827/0236-235X.123.598-604 (in Russ.).

\section{Для цитирования}

Игнатьев С.В., Красников А.В., Осипов А.А., Шаталина Н.В. Модемь формирования операционных знаний дмя экспертной системы обеспечения запасными частями, инструментами и принадмежностями радиоэлектронных средств // Программные продукты и системы. 2021. T. 34. № 4. C. 617-628. DOI: 10.15827/0236-235X.136.617-628.

\section{For citation}

Ignatev S.V., Krasnikov A.V., Osipov A.A., Shatalina N.V. A model for the forming operational knowledge for an expert system for providing radioelectronic facilities with spare parts, tools and accessories. Software \& Systems, 2021, vol. 34, no. 4, pp. 617-628 (in Russ.). DOI: 10.15827/0236-235X.136.617-628. 